\title{
Managing the Shift in the Enterprise Perimeter in order to delay a Cybersecurity Breach
}

\author{
Muyowa Mutemwa \\ Council for Scientific and Industrial \\ Research \\ Pretoria, Gauteng, South Africa \\ mmutemwa@csir.co.za
}

\author{
Mfundo Glenn Masango \\ Council for Scientific and Industrial \\ Research \\ Pretoria, Gauteng, South Africa \\ MMasango1@csir.co.za
}

\author{
Dr Noluxolo Gcaza \\ Council for Scientific and Industrial \\ Research \\ Pretoria, Gauteng, South Africa \\ ngcaza@csir.co.za
}

\begin{abstract}
In the year, 2019, South African enterprises have seen a substantial increase in the number of cybersecurity incidents. From the public-sector to the private-sector, many enterprises have had to redirect large sums of their budgets towards responding to cybersecurity incidents that could have been averted. For most enterprises, having experienced, or seen the impact of a number of disclosed cybersecurity incidents in South Africa (SA) during 2019, the majority of enterprises had begun to strengthen their cybersecurity defenses in order to avert future cybersecurity incidents. To a large extent, most enterprises have implemented security measures such as firewalls, proxy gateways, patch management solutions, intrusions prevention or detection systems, encryption, network access control and so on. However, whilst these measures are implemented, in 2020, security architects saw a shift in the enterprise perimeter due to COVID-19 pandemic. Owing to the pandemic, on the 26th of March 2020, the South African government implemented a countrywide lock-down effectively enforcing all its citizens to stay at homes thus translating to an unintended remote workforce. During this time, employees found themselves overnight without any training working outside of the secured layered cybersecurity defenses of their enterprises. This arrangement negatively affected the security posture of many organizations. It effectively forced all enterprise employees to work from home, away from the secure enterprise network forcing information technology administrators to find ways to extend the enterprise perimeter in order to allow these inexperienced overnight remote workers to continue to securely work remotely. This article explores the consequences of the
\end{abstract}

Permission to make digital or hard copies of all or part of this work for personal or classroom use is granted without fee provided that copies are not made or distributed for profit or commercial advantage and that copies bear this notice and the full citation on the first page. Copyrights for components of this work owned by others than the author(s) must be honored. Abstracting with credit is permitted. To copy otherwise, or republish, to post on servers or to redistribute to lists, requires prior specific permission and/or a fee. Request permissions from permissions@acm.org. icARTi '21, December 9-10, 2021, Virtual Event, Mauritius

(c) 2021 Copyright held by the owner/author(s). Publication rights licensed to ACM.

ACM ISBN 978-1-4503-8575-6/21/12 ..\$15.00

https://doi.org/10.1145/3487923.3487925 shift in the enterprise perimeter and also suggests practical technical steps that enterprises can apply in order to avert possible cybersecurity incidents.

CCS Concepts: - Security and privacy $\rightarrow$ Intrusion/anomaly detection and malware mitigation; Intrusion detection systems.

Keywords: Work from Home, COVID-19, Enterprise Perimeter, Malware, and Phishing

\section{ACM Reference Format:}

Muyowa Mutemwa, Mfundo Glenn Masango, and Dr Noluxolo Gcaza. 2021. Managing the Shift in the Enterprise Perimeter in order to delay a Cybersecurity Breach. In International Conference on Artificial Intelligence and its Applications (icARTi '21), December 9-10, 2021, Virtual Event, Mauritius. ACM, New York, NY, USA, 10 pages. https://doi.org/10.1145/3487923.3487925

\section{Introduction}

Successful breaches that occur due to an exploit of human behaviour are on the increase. This article begins with a literature review from within South Africa and then from around the world of cybersecurity incidents that benefitted from some sort of human interaction. The literature review is key to understanding the impact and magnitude of a successful breach due to the exploit of human behaviour through social engineering.

According to the 2020, annually released Data Breach Investigation Report published by Verizon [2], the top three causes of breaches which contributed to 67 percent of the total breaches were because of credential theft; social attacks from phishing and business email compromises; and human error. From the report, it can be seen that humans are a direct or indirect target for threat actors.

\subsection{Prominent South African based Cybersecurity Breaches}

In July 2019, City Power, an energy utility in South Africa, was compromised by a Ransomware attack which left its customers unable to purchase electricity units and unable to visit the group's website [26]. According to City Power, the ransomware was able to encrypt all internal databases and applications [4]. In October 2019, the City of Johannesburg systems were compromised by a group called "Shadow 
Kill Hackers" who claimed they had access to all servers' data including all passwords and personal employee information and they also claimed they had multiple back doors into all computer systems [14]. The hackers demanded 4.0 bitcoins be paid into a bitcoin wallet in order to remove their backdoors and disclose their methods of attack. In the same year, 2019, according to the South African Banking Risk Information Centre (SABRIC) [20], on the 23rd of October, South Africa banks experienced a wave of ransom driven Distributed Denial of Service (DDoS) attacks. This wave of attacks began with a ransom note that was delivered via emails to both unattended mailboxes as well as staff mailboxes publically listed on websites. According to SABRIC, this type of attack did not involve hacking or a data breach, but rather increased traffic on the bank networks necessary to access public facing services and therefore no customer data is at risk.

\subsection{Prominent World Wide Cybersecurity Breaches}

2020 saw an increase in the number of cybersecurity incidents from large organisation to small organisations. Of note, Twitter's verified user accounts were compromised on the 15th of July 2020 [25]. According to TheMotherboard [5], they believe that a Twitter user administration tool was accessed by the hackers after images of the administrative tool surfaced underground. At the time of writing, it is believed that the attackers gained access to the administrative tool through a social engineering attack. According to Twitter [25], the hackers were able to access the user profiles of verified accounts such as Barack Obama, Joe Biden, Elon Musk, Bill Gates, Uber, and Apple using a coordinated social engineering attack on Twitter employees which led to the successful access to Twitter's internal systems and tools by the hackers. The Hackers then further used a social engineering bitcoin scam through tweets sent from these verified accounts to solicit followers of these verified Twitter accounts to deposit bitcoin into a bitcoin wallet. The Twitter incident shows that a successful social engineering attack can evade mechanisms such as multifactor authentication(MFA).

On the 23rd of July 2020, Garmin Ltd. released a press statement stating it had become a victim of a cyber-attack that encrypted some of our systems [8]. According to the same Garmin press statement, Garmin stated that although its online services were affected including website functions; customer support; customer facing applications; and company communications there was no indication that any customer data had been stolen or accessed.

\subsection{Cybersecurity as a Threat to Organisational Growth}

On a global scale, chief executive officers' responded to the PricewaterhouseCoopers survey of 2019, and highlighted cybersecurity threats as the fifth biggest threat to the growth and development of their organisations [17]. Also according PricewaterhouseCoopers [18], survey of 2019, responders viewed cybercrime as the fourth most disruptive economic factor to the growth of South African enterprises in the next 24 months. Having seen some of the cyber incidents from 2019 in section 1.2, it is no surprise that cybercrime is listed so high up by the survey responders. The responders of the PricewaterhouseCoopers survey [18], stated that disruptive economic cybercrimes towards South African Enterprises were perpetrated mostly by internal personnel with $41 \%$; followed by external personnel with $36 \%$; and collusion of both internal and external with $21 \%$. PricewaterhouseCoopers consider cybercrimes involving senior management as insider perpetrators to be among the most harmful because of the senior management's ability to override or bypass governance. It is easy to conclude that a successful social engineering attack involving impersonation of senior management could yield the same results of bypassing or overriding governance.

This article aims to look at the cybersecurity challenges of extending the cooperate network perimeter by introducing remote workers through the analysis of a case study. Following the challenges, article then aims to present recommendations that organisation can implement to deter a cybersecurity breach. The rest of the article is structured as follows. Section 2 presents a case study to explore some of the cybersecurity challenges employees face in a pandemic era while working remotely. Section 3 looks at why attacks use interactive attacks that require human input and the objectives the attacks hope to achieve. Section 4 presents recommendations that organisations should implement in order to delay a cybersecurity breach that is introduced remotely on a user's device and then propagated onto the cooperate network.

\section{Environmental Case Study}

This sections details results that were obtained from a case study. The case study covers threats that a single South African organisation's employees faced during the lockdown period of July and August 2020. While the employees were working from home, it was possible to collect directory service logs, antimalware logs, Domain Name Service (DNS) resolution logs and email gateway logs, from the users' machines. The users' machines have Security Information and Event Management (SIEM) agents installed on them and logs were sent back to the SIEM tool on the cooperate network over the internet through encrypted secure protocol. The pie-charts and tables presented in this section are a summary of the malicious incidents experienced by employees on their laptops while working from home, during the months of July and August 2020. The organisation used for the case study had at the time of writing this article, 8000 employees working from home. From the SIEM logs collected and analysed, 43 percent of the employees had at least had either a failed 
logon that is a windows event code of 4625 or successful logon that is windows event code of 4624 . This means that from the security logs collected and analysed by the security operations centre from which the highlights in this section are obtained shows that 3440 employees active during this period. The remaining 57 percent of employees were inactive that is they did not switching on their laptops. It is also possible that, that the SIEM agents responsible for collecting the security events from the employees' laptops were not functioning during those months. It was not possible to route all user machine traffic through the cooperate firewalls, proxy servers, and other cooperate security devices found on the cooperate network, therefore these devices are left out in this case study. The reason for this case study was to, firstly look at user behaviour, secondly look at the malware that was found on user machines, thirdly look at the website requests made by users on their machines, and lastly analyse malware signatures found in emails.

In the subsection 2.1, the article looks at the reasons why users had failed logins while working from home. In the subsection 2.2, the article looks at how ransomware can be introduced into the cooperate network from a remote user's device. In the subsection 2.3, the article looks at the IP addresses infected machines requested. In the subsection 2.4, the article looks at how malware can be introduced onto a remote user's device through unsolicited malicious emails. This case study is important in understanding the cybersecurity challenges that remote users face while working remotely away from the cooperate office network's proven layered security defenses which provide protection and automated response.

\subsection{Directory User Behavior Analysis}

The logins mentioned herein this subsection were local logins into the physical user machines, virtual private network (VPN) logins and remote web email login.

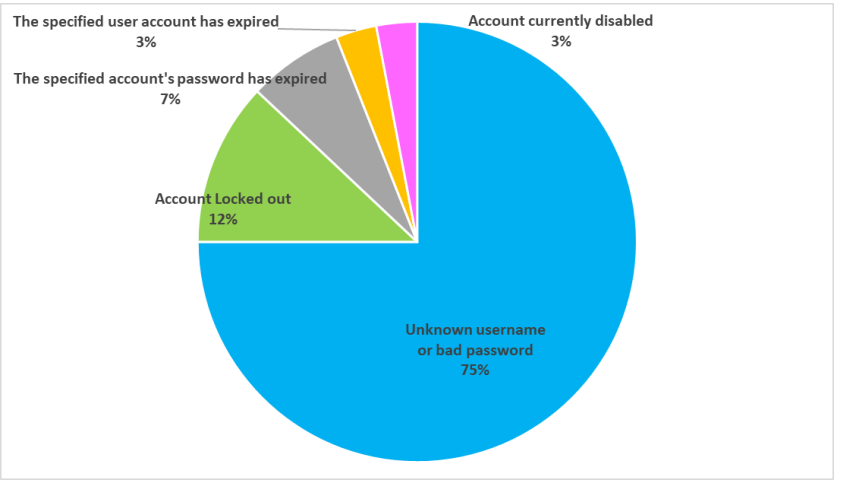

Figure 1. User Behavior Analysis

Although the errors were more descriptive on the SIEM tool, the remote users could only see standard logon errors displayed by the local machine, VPN client, or web email portal. No additional information was provided to the remote user, except when users logged a call with the service desk. Figure 1 shows the top five reasons why users were not able to logon to the local machines, VPN client or web email. This subsection is important because from it, an understanding can be drawn as to why remote users would have failed login attempts.

Firstly, the majority of users could not log onto their accounts because the username or password was incorrect. Secondly, there were user accounts that were locked out because the threshold of 10 failed attempts were reached. Any further subsequent attempts to logon after the threshold was reached resulted in the "account locked out" logon error. Thirdly, there were user accounts that had passwords older than 60 days. The password expiry policy was adjusted from 30 days to 60 days to reduce the number of service desk calls during the entire lockdown period. Any user attempts to logon with a password older 60 days resulted in "the specific account's logon password has expired" logon error. Fourthly, if an attempt was made to log with a user account of employee who had exited the organization, the "accounts currently disabled" logon errors was observed. The fourth logon error was mostly seen on the web based email portal. Lastly, if an attempt was made to logon with a user account of contractors with specific account expiry date and the logon attempt was after the specified date, "the specific user account has expired" logon error was observed.

A closer analysis of the output leads to two main conclusions. The first is that remote users save or cache their credentials in order to make their life easier. This means that when they access services through a web portal after a password change or reset, the login is made with an old password thus there will be an increase in failed logins attempts which lead to an account lockout. The second is that the high number of failed logins were because of a malicious application or process running locally on the remote user device that attempts to authenticate using the remote user's credentials.

\subsection{Antimalware Analysis}

The malware results mentioned herein in this subsection were obtained from the antimalware software installed on all user machines including contractors. The antimalware software was able to update its signatures remotely as long as the user had an internet connection. The antimalware performed one aggressive scan a week and daily quick scans. This subsection is important because from it, an understanding can be drawn as to malware that users could encounter while working remotely and the challenges that could occur when anti-malware software attempt to perform remediatory actions against detected malware.

Figure 2 shows the top five-malware signatures that were found and blocked by the antimalware software. From Figure 
2 , it can be seen that the most observed malicious signature found on the remote user machines was the "Trojan.Gen.2" signature, followed by the "W32.Virut.CF" signature and the "Heur.AdvML.B" signature. According to [28], "Trojan.Gen.2" is a computer Trojan that once it has infected a user's machine, this Trojan is able to establish an outgoing internet connection to a remote $\mathrm{C} 2$ server to elicit further instructions.

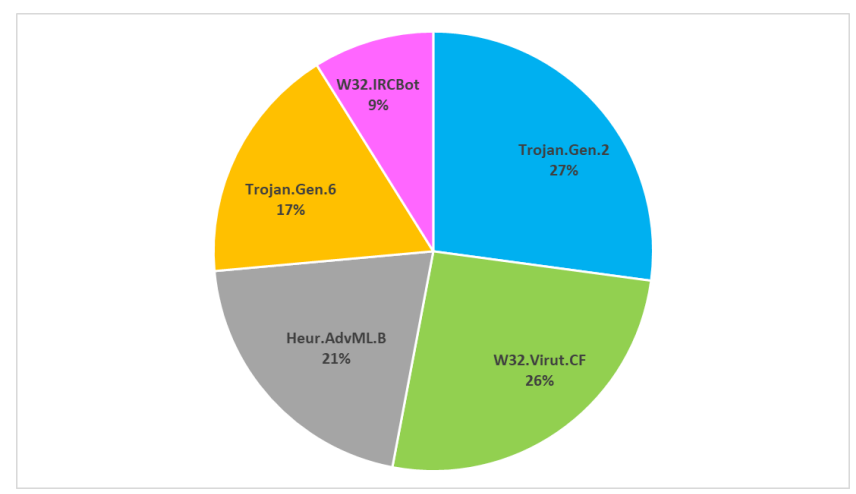

Figure 2. User Behavior Analysis

According to [7], "W32.Virut.CF" is a virus that infects executable files such as "EXE", "SCR" and other Portable Executable (PE) file formats. The "W32.Virut.CF" is also able to inject an iframe into the body of the web page files such as "HTML", "PHP" and "ASP" which can be effective for social engineering attacks.

According to [9], "Heur.AdvML.B" is an Advertisingsupported Software (Adware) that can be installed directly on the user's machine or as a browser extension. Adware is unwanted software designed to automatically play, display or download advertisements on a user's machine, in most cases within a web browser. Although some adware are harmless, other adware have been classified as Privacyinvasive Software (Spyware). In this case, "Heur.AdvML.B", commonly known as "DealBeaver Ads" is adware supported and promoted by DealBeaver.org. According to DealBeaver, the "DealBeaver Ads" adware is supposed to help users find interesting deals, vouchers, and coupons from online stores such as Amazon, AliExpress and so on. However, according to [9] [16] "Heur.AdvML.B" is capable of numerous malicious actions such as tracking web cookies, personal user information theft, record a user's keystrokes together with the sites they visited, click fraud, and so on.

The malicious signatures from Figure 2 were mostly found in the following top seven filenames namely " 3 Months Contracts Admin \& General 2018.xls.exe", "Liehfweih.exe", "autorun.inf.exe", "Sharon COVER NOTE.exe" "Desktop.ini.exe", "Counter-Striker.exe" and "DWH4266.EXE". Figure 3 shows the top five actions automatic performed by the remote antimalware agent once the malicious signatures from Figure 2 were detected. The actions in Figure 3 did not require manual interventions from the IT service desk resolver in order to remediate.

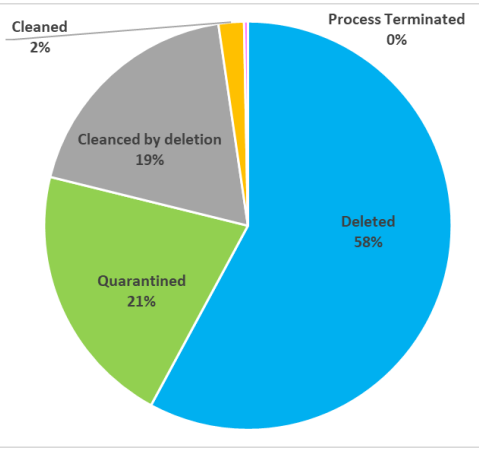

Figure 3. User Behavior Analysis

However, a few actions did require manual interventions. These actions are listed in Table 1 . For each of the cases in Table 1 an analysis was perform on the infected file to determine:

- the file location (a system file, library, installed application, user file, temp file or external file on a USB drive);

- what the file is used for;

- and the impact of removing the file.

Table 1. Antimalware Action

\begin{tabular}{cc}
\hline Antimalware Action & Event Occurrence \\
\hline Left Alone & 14 \\
Pending Repair & 6 \\
Partially Repaired & 3 \\
Moved back & 2 \\
Process terminated pending restart & 2 \\
All actions failed & 2 \\
Access Denied & 1 \\
\hline
\end{tabular}

For "Left alone", "Moved Back", "All Actions Failed" and "Access Denied" actions, the files were either "user files" or "temp file". Thus, an IT service call was logged to issue a "Power Eraser command" to forcefully remove the files through a command on the antimalware management platform on the cooperate network to the anti-malware agent on the remote user's machine and thereafter perform a rescan. For the "Pending Repair" and "Partially Repaired" actions, if the files were "installed applications" then the applications were uninstalled, the remote machine was rescanned and the application was reinstalled. The application was reinstalled provide, it served a business function. If the files were "temp files" or "external files on USB drive" then a "Power Eraser command" was also issued to forcefully delete the files. Files 
found on external USB drives were difficult to erase, especially where users had removed the USB external drive. If the files were "system files", the IT service desk resolver's actions would have to physically drive to the remote user and obtain the machine, perform a user files backup, reinstall the operating system, restore backup files and then drive back to the remote user to hand back their machine. System file(s) infections took, on average six working days to resolve.

For the "Process terminated pending restart", an IT service desk call was logged to forcefully restart the user's machine through the Microsoft System Center Configuration Manager (SCCM) issued command or policy. Once a restart was completed, the remote user's machine was rescanned.

One of the major challenges observed with action items from Table 1 was that the remote antimalware agent could only reciprocate the action from the antimalware management platform once the remote user's machine was active and connected to the internet. Remote users were not always connected to the internet, thus in some cases the antimalware updates were not always applied, and antimalware logs were only received once the user machine was connected to an internet connection. The same applied for the Microsoft SCCM agents. In some cases, the antimalware software detected ransomware files on the remote users' machines. Table 2 shows the sample ransomware signatures and files paths. From Table 2 it can be seen that there are two ransomware signatures namely "Ransom.Crysis" and "Ransom.Wannacry".

From Table 2 it can be seen that the sample ransomware files were introduced on the remote user's machine through the computer drive letters D, E, F and H since the case study organisation defaults the operating system installation to the $\mathrm{C}$ drive on a computer. Thus, in all cases, the ransomware was introduced onto the remote user' machine through an external USB drive or external storage. From Table 2 it can also be seen that the files are entertainment files, such as movies or television shows. This means that as remote users plugged-in external drives to watch movies or television shows on their work laptops they were without realizing it introducing ransomware onto the cooperate laptops. The take away is that not only ransomware but also all malware can be introduced onto a remote user device through external drives.

\subsection{Type of Blocked DNS Requests}

The DNS results mentioned herein in this subsection were obtained from the cloud-based DNS servers logs. This subsection is important because from it, an understanding can be drawn as to the type of malicious DNS requests a user device could be making while employees are working remotely. This section also stresses the importance of a cloud-based DNS server that can be reached by remote user machines. All employee user machines, including contractors and service providers have their machines' DNS resolver pointed to the cloud-based DNS server. Thus all DNS queries both on the cooperate network and the remote workers will be resolved by the same set of cloud-based DNS servers.

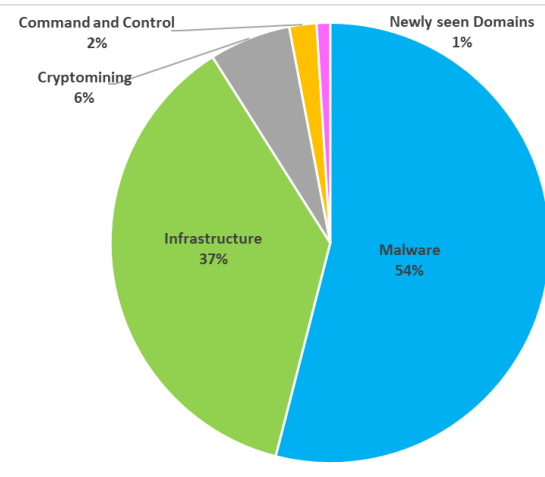

Figure 4. Top 5 Blocked URL Categories

From Figure 4 it can be seen that the top five blocked DNS requests were for "Malware", "Infrastructure", "Cryptomining", "Command and Control (C2)" and "Newly seen Domains". The cloud-based DNS server responds to all malicious domain requests on the DNS blocked list with a sinkhole IP address. The DNS sinkhole means that the DNS forwarder will return a false IP address to a requested malicious URL. Thus, the sinkhole prevents access of malicious URLs within the organisation.

In Table 3, the "occurrence percentage" is based on all blocked DNS requests. In Table 4, the "occurrence percentage" are only from the "C2" category. If the "C2" category were to be added to Figure 5, the occurrence percentage in comparison to other categories would be less $1 \%$. Thus in Table 4 the "occurrence percentage" in the fourth column is based only on URLs related to the "C2" category.

According to Cisco Umbrella, the "pc.pc0416.xyz" domain in Table 3 is blocked by the Cisco security features because the domain contains malware, while the "gulf.moneroocean.stream" domain is associated with cryptocurrency mining.

In cases where the user machines attempted to access known C2 server URLs, the remediation was to log an IT service desk call to verify that the remote antimalware agents were up to date, initiate a remote "aggressively scan" on the user machines and keep the machines on a watch list. If a user machine again attempted to contact a C2 server, the remediation was to log a call with the IT service desk for a resolver to use the authorized software to create a remote connection to the user machine over the internet through a secure remote desktop management software and then uninstall browser tools or extensions. The case study organization has a least privilege policy in place, which ensures that no remote users can install or uninstall any software on their cooperate machine. Software installation is pushed to remote users through the Microsoft SCCM agent on the 
Table 2. Sample Ransomware Signatures and File Paths

\begin{tabular}{|c|c|}
\hline Signature Name & File Path \\
\hline Ransom.Crysis & E:IStar Wars The Rise Of Skywalker (2019) [720p] [WEBRip] [YTS.MX]|Subs.exe \\
\hline Ransom.Crysis & F:|The Grudge (2020) [720p] [WEBRip] [YTS.MX]|The Grudge (2020) [720p] [WEBRip] [YTS.MX].exe \\
\hline Ransom.Crysis & D:IJumanji.The.Next.Level.2019.HC.720p.HDRip.800MB.x264-GalaxyRG[TGx].exe \\
\hline Ransom.Crysis & D:|Frozen II (2019) [720p] [WEBRip] [YTS.MX].exe \\
\hline Ransom.Crysis & D:|Bad.Boys.for.Life.2020.720p.WEBRip.900MB.x264-GalaxyRG[TGx].exe \\
\hline Ransom.Crysis & F:|The.Witcher.S01.COMPLETE.720p.NF.WEBRip.x264-GalaxyTV[TGx].exe \\
\hline Ransom.Crysis & D:|6 Underground (2019) [WEBRip] [720p] [YTS.LT].exe \\
\hline Ransom.Wannacry & $\mathbf{H}: \mid$ Users $\backslash$ Administrator $\backslash A p p D a t a|L o c a l \backslash T e m p \backslash p c t| v 0 j . e x e$ \\
\hline Ransom.Wannacry & $\mathbf{H}: \mid$ Users $\backslash$ Administrator $\backslash A p p D a t a \backslash$ Local $\backslash$ Temp $\backslash$ pct $\mid v 0 j . e x e$ \\
\hline
\end{tabular}

Table 3. Top 5 Most Blocked URLs

\begin{tabular}{llcc}
\hline Blocked URL & Category & Occurrence Percentage & Risk Rating \\
\hline pc.pc0416.xyz & Malware & $54 \%$ & High \\
gulf.moneroocean.stream & Cryptomining & $37 \%$ & Low \\
takkj2016.redirectme.net & Dynamic DNS & $6 \%$ & Medium \\
epicunitscan.info & Potentially Harmful & $2 \%$ & Low \\
v1.litcsxdj.website & N/A & $1 \%$ & Low \\
\hline
\end{tabular}

user's machine. As a result there was no concern that install applications were contacting known $\mathrm{C} 2$ servers.

A sample of "C2" blocked DNS requests can be seen in Table 4. From Table 4 the "gvaq70s7he.ru", "v1.gboxzxlh.mobi" and "wmi.my0709.xyz" domains in Table 4 are blocked due to their association with Botnet activity while the "differentia.ru" and the "disorderstatus.ru" from Table 4 are highly likely to be Fast flux domains.

A closer analysis of the output leads to two main conclusions. The first is it is important to monitor the DNS requests that are made by remote devices especially blocked DNS requests for web resources. The second is that a high number of blocked DNS requests came from an installed malicious browser tools, extensions or adware that were installed without the remote user's knowledge or permission.

\subsection{Blocked Malicious Signatures in Emails}

The malicious email signature results mentioned herein in this subsection were obtained from the cloud-based email gateway logs. All emails, outbound and inbound; and between mailboxes within the cooperate network have to traverse the email gateway. As the emails traverse the gateway, each email is filtered for SPAM; scanned for phishing links or attachments; and scanned for malware signatures. This subsection is important because from it, an understanding can be drawn as to the type of malicious emails that employees could be receiving from attackers in an attempt to compromise the remote device or obtain the remote user's cooperate credentials.

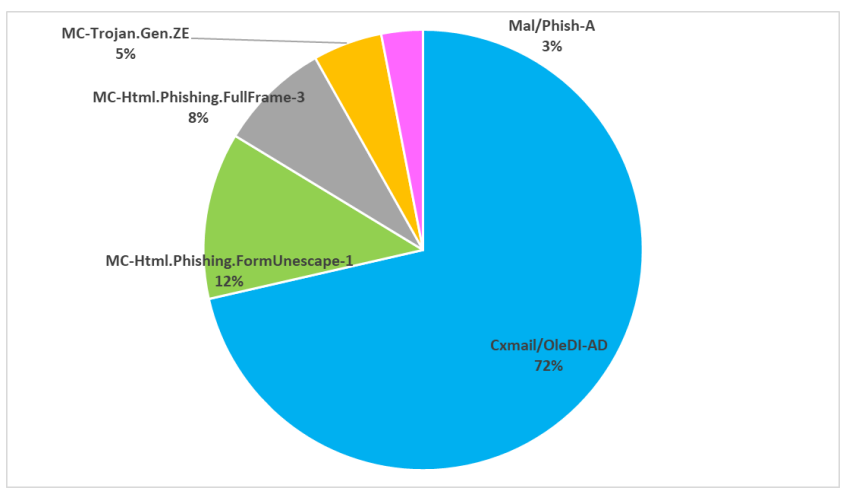

Figure 5. Blocked Malicious Signatures in Emails

Figure 5 shows the top five malicious signatures observed in emails. From Figure 5 it can be seen that the "Cxmail/OleDI-AD" was the most common malicious signature followed by "MC-Html.Phishing.FormUnescape-1" and "MC-Html.Phishing.FullFrame-3". According to [22], "Cxmail/OleDI-AD" is either a virus or spyware found within a Microsoft Word document 95 to 2003.

A closer analysis of the output leads to two main conclusions. The first is that users will continue to receive malicious emails whether they are working on the cooperate network or remotely. The second is, it is important to monitor malicious email signatures to understand the threats that remote users face. 
Table 4. Top 5 Blocked DNS C2 URLs

\begin{tabular}{llcc}
\hline Blocked URL & Activity & Occurrence Percentage & Risk Rating \\
\hline gvaq70s7he.ru & Botnet (Kelihos) & $49 \%$ & High \\
v1.gboxzxlh.mobi & Botnet RAT & $39 \%$ & High \\
wmi.my0709.xyz & Botnet RAT & $6 \%$ & High \\
differentia.ru & Botnet (Andromeda) & $4 \%$ & High \\
disorderstatus.ru & Botnet (Andromeda) & $2 \%$ & High \\
\hline
\end{tabular}

\subsection{Case Study Summary}

From the case study it can be seen that it important to have visibility and detailed insight as to why user logons are failing remotely. The case also shows the importance of having visibility to type of malware, users face remotely, what IP addresses the malware will attempt to access and malicious unsolicited emails that attackers send to employees while they are working remotely. It is also important to have visibility to determine if remote user devices are infected with Adware or Spyware.

In the following section, the article explores different techniques that attackers use in order to materials the incidents and attacks presented in this section.

\section{How Attackers Use Malware}

In the case study, the article presented the different types of threats users faced while working remotely. In this section, the article will present why threat actors prefer to use these threat vectors and what they hope to accomplish. There are four ways and reasons why attackers make use of malware for remote users. Firstly, for exploited assets to communicate back to the $\mathrm{C} 2$ server, secondly to download and execute ransomware payloads, thirdly to spy on user activity through spyware or key loggers, and lastly cryptocurrency mining. The following subsections will discuss these approaches in detail.

\subsection{Command and Control (C2)}

Botnets are a collection of infected machines that operate in an automated manner based on instructions they receive from a $\mathrm{C} 2$ server.

The first stage is to get the user's machine to receive the bot binary through a number of propagation vectors such as downloading and installing unverified software; clicking on malicious links in emails; opening malicious documents; posting of malicious links on social media; redirects to malicious websites; accessing USB external drives with malware; and so on. The bot binary is an executable code that be directly executed by a user machine without requiring any user interaction. The second stage is to get the user' machine to execute the bot binary. Once the bot binary is successfully executed without prohibition or detection, the third stage is to get the infected machine to contact the $\mathrm{C} 2$ server in attempt to enlist in the botnet. This initial contact to the $\mathrm{C} 2$ server is known as rallying. Rallying can be through a seedlist of IP addresses of other botnets that know how to contact the $\mathrm{C} 2$ server; a hard coded IP address or Fully Qualified Domain Name (FQDN); and so on. In the fourth stage, once the user machine is part of the botnet, it can then receive instructions and execute commands. The fifth stage is where the attacker would repeat stages one to four in order to recruit more machines to join the botnet. Fast flux is a DNS technique used by botnets to hide phishing and malware delivery sites behind an ever-changing network of compromised machines. These compromised machines act as proxies to obscure the true IP address of a malicious site. The main idea for fast flux is to swap in and out multiple IP addresses associated with a single FQDN related to the $\mathrm{C} 2$ server.

\subsection{Ransomware}

From the 2020 Data Breach Investigation Report, 27 percent of malware incidents involved ransomware [1]. It was also reported that 18 percent of organisation blocked at least one incident of ransomware. According to Sophos [23], there are four stages that attackers employ to execute a successful ransomware campaign. The first stage is to deliver the ransomware onto the victim's machine. This is done through SPAM email attachments or directing a user to visit a compromised website. The second stage is for ransomware to install and collect information about the victim's machine vulnerabilities. It is in this stage that the information that is collected is sent back to the $\mathrm{C} 2$ server through a secure port. In the third stages, the $\mathrm{C} 2$ server will send specially crafted payload(s) to the victim's machine together with the encryption keys. During this stage, the victim's machine is encrypted, including network drives and if possible, the ransomware spreads to other machines on the network. In the final stage, the ransomware will delete the keys and itself. A ransom note is then displayed.

\subsection{Spyware}

Spyware is software that is designed to collect sensitive user's information from a user's machine [10]. This information is then sent to an attacker who has malicious intent. Spyware can also manipulate search results by substituting legitimate search results with malicious URLs that victims 
would then click on. Spyware can be introduced onto a user's machine through a Trojan, web tools or extensions, compromised tracking cookies, and so on. Spyware can be used to log keystrokes on a computer keyboard; use the microphone, location, camera and so on to spy on user activity; degrade user machine performance; and code execution. Spyware interacts with the web browser are invoked in response to events by the browser functions.

\subsection{CryptoJacking}

Developers of online services especially free-online services delivered to the end-user via the web browsers are considering a shift from advertisement-based revenue income to web-based cryptography (crypto) currency mining [19]. Webbased crypto currency mining is being seen as a real revenue model for web operations and developers. In web-based crypto mining, the idea is to solve computationally expensive puzzles as a proof of work where a new block is discovered and linked to the previous block in the blockchain. With each discovery or link, currency is awarded however, the mining difficulty rises. In order to balance the increased difficulty, developers must increase their distributed mining network to get access to more computational power.

With web-based crypto mining, the web developers are able to utilize each visitors' CPU resources during the visit through embedded mining code found on their website. However, cryptojacking, that is mining without user's consent poses a significant challenge and it is a known cybersecurity attack vector.

\subsection{Section Summary}

Section 3 looked at four reasons why a threat would like to compromise a remote user machine with malware. The first reason is to invite the remote user's machine to join the threat actor's botnet. The second reason is make the remote user machine become the medium through which ransomware is introduced onto a cooperate network. The third reason is to spy on sensitive users activities performed on infected cooperate devices. The last reason is to utilize the remote user machine's resource in mining cryptocurrency.

In the next section, the authors share 10 recommendations on how organisations can reinforce the cybersecurity posture of the extended cooperate security perimeter for remote users to delay the threats herein mentioned.

\section{Recommendations}

From the case study in section 2 and the analysis of how attackers make use malware in section 3, the authors of this article recommend the following multilevel approach actions for organisations to implement in order to delay impending lockdown related cybersecurity breaches, which would arise from a successful social engineering campaign or malware execution.

\subsection{Adherence to strict Governance}

PricewaterhouseCoopers considers that Enterprises place greater focus on governance [18]. Ensuring that is there are cybersecurity frameworks, standards procedure, and policies. The procedures and policies should be tested to ensure that they are relevant and updated. The procedures and policies should be specific as to define roles, reporting hierarchy, impact, recovery steps, and so on.

\subsection{Employees Cybersecurity Awareness}

Employees and third party users should be educated with contextually relevant cybersecurity awareness training applicable to their private life, work, department and the organisation. The first step is educating users on how to ensure that their home networks and online accounts have good security such as changing the home router's default credentials; use of password managers instead of reusing passwords; updating and patching software on devices; securing their internet baking and online shopping; not clicking on links especial social media; and so on. The second step is for employees to have simulated scenario-based training based on their everyday functions and the tools they used for work. The third step is to have performance indicators to measure success. The four step is to provide feedback to their employees and third party users on their likelihood to be susceptible to attacks.

\subsection{FA for Authentication}

Two-factor Authentication should be enabled for authentication to email, online services, laptops, servers, and so on. Although the addition of a secondary authentication factor is not a silver bullet for verifying authentication, it does however add a deterrent layer to the defence-in-depth approach. Two-factor authentication can prevent unauthorized logins using saved credentials such as those stored by browsers; brute-force attacks; reuse of harvests credentials; and so on [13].

\subsection{Authorized User Behaviour Monitoring and Logging}

User behaviour should be analysed for the following three reasons, namely the first external attackers can compromise internal user accounts in order to achieve their target of a successful breach. Secondly, employees or third parties as authorized users can attack an organisation and become malicious insiders. Thirdly, employees can mistakenly cause harm or create a vulnerability through human error. Thus, all authorized user activity at all levels including those of contractors or partners should be logged; perform a periodic review of user access rights; and user accounts that are inactive for more than 30 days should be disabled. 


\subsection{Disable USB ports for External Storage}

For the organisation in the case study, it is evident that malware was introduced onto the cooperate devices through external USB storage drives. The most effective way to prevent known malware signatures and zero-day attacks emanating from external USB drives is to first assess the business impact and thereafter disable the use of USB ports for external storage devices through the directive service policy such Microsoft Group Policy [6].

\subsection{Cloud-Based DNS Resolution}

For organisation that have their DNS servers on their cooperate network. These organisations should consider cloudbased DNS servers that can be reached by the devices of the remote workers. Cloud-based DNS services are able to aid in the protection of users of an organisation, irrespective of the fact that the user is on the cooperate network or working from home [21]. The administrators of these cloud-based DNS servers should be able to configure security measures such as DNS sinkholes, identification of the user and device name; audit logs, and so on. In cases where an organisation does not have a budget for a cloud-based DNS service, consideration should be made for free DNS services. However there are drawbacks to free DNS services such as a possible lack of viability, lack of reporting, lack of support, lack of audit logs, and so on.

\subsection{Cloud-Based Email Gateway}

As mentioned previously, email gateways are able to filter inbound, outbound, inter-mailbox exchange of emails. Among other features, email gateways are able to filter out SPAM, dangerous file types, malicious attachments and impersonation attacks. The implementation of a cloud-based email gateway will reduce the amount of malicious emails, links or attachments that employees and third party users have to interact with. The email gateway will also provide visibility through logs obtained from the email metadata the attacks targeted towards your organisation and the most targeted mailboxes.

\subsection{Updates, Security Patches and Policies}

Although applying security updates and patches remains one of the completex operations performed by the technology administrators of systems within an organisation, it remains important to ensure that all software within the cooperate IT environment are regularly updated including the antimalware software [1]. The challenge comes when users are no longer in the IT environment and they are on their home network with other devices locally including Internet of Things (IoT) that have not been updated or could have been previously compromised. The first step for organisations is being able to push down operating system security patches, application patches, and so on. This also applies to
Microsoft SCCM. The second step is being able to continue to push down antimalware signatures signature updates to the remote users' machines that have an internet connection. The third step is being able to pushdown user, machine or profile policies using secure protocol to the remote machines. The fourth step is being able to log all antimalware activities such as infections, scans, attacks, and so on.

\subsection{Directory Services Administrative Tier-ing}

According to Microsoft [12], directory service tiering model adds another layer to the defense-in-depth security approach. Microsoft proposes a three Tiering model for administrative accounts, this tiering model does not include standard user accounts, machine accounts or service accounts. The Tiers are namely Tier 0 , Tier 1 and Tier 2 . The administrators in Tier- 0 are only allowed to have control over enterprise identities in the environment. The administrators in Tier-1 are only allowed control of the enterprise servers and application on a server and application basis. The administrators in Tier-2 are only allows control on user devices. All others users should be given least privilege rights sufficient to perform their daily duties. All user activity within the enterprise should be logged and monitored. Administrator rights in each of the Tiers should be periodically reviewed.

\subsection{Continue to Receive Audit Logs}

Most organisations already have audit logs enabled on their employee devices and in some cases including third party partners or service providers. The logs on a remote machine would include user behaviour actions, permissions, antimalware logs, host-based intrusion detection or prevention system logs, and so on [15]. The first step would be ensure that there is accurate visibility that ensure that these security layers are still functioning correctly on the remote devices. The second step would be ensure that these systems are still logging. The third step would be ensure that the logs can send from the remote devices into the cooperate network through a secure protocol in most cases particular to the SIEM agent installed on the remote user device. The fourth step would be adjust and then establish a new baseline for users behavioural as most users would log into their machine or work at odd hours of the 24 hour day.

\subsection{Artificial Intelligence enabled Security Controls}

The case study organisation used in this article indexes on a daily basis 500 gigabytes worth of logs from different endpoint-based and network-based security systems. In general this high number security logs indexed and analysed daily would cause cybersecurity analysts to be overwhelmed with the high number of security events. Traditional tools that only depend on signatures and rules to block cybersecurity threats are no longer sufficiently equipment to protect against new cybersecurity threats emanating from advanced 
persistent threats and zero-day attacks [11]. Artificial intelligence can be applied to the detection, response and recovery from the high number of cybersecurity incidents [24]. In particular, machine learning can be used to provide a reliable phishing email filter that is based on the authorship of cybersecurity threats found in the phishing emails [3]. Security tools that make use of artificial intelligence will reduce the amount of false positives, incidents investigated by cybersecurity analysts and help automate actionable steps. Thus it is important to upgrade to or deploy artificial intelligence enabled security tools to ensure that cybersecurity analysts are not outpaced by the number of incidents [27]. The biggest problem in cybersecurity is not always the failure to add more security tools as sensors for detection but how to enable the analyst to keep apace with the sheer volume of alerts being generated. However, the deployment of artificial intelligence enabled security tools is not a silver bullet because like any security control they too are subject to the occasional failures [27]. In conclusion, artificial intelligence enabled security tools must be added to the layered defence of security tools.

\section{Conclusion}

This article looked at the recent cybersecurity attacks in South African and across the world. A case study was covered in this article in order to understand the threat vectors used by criminals to attack remote machines during the Covid19 work from home. The article closes with recommendations that if organisation are already not implementing should implement. The implementation of these recommendations will ensure that an organisation delays the possibility of experiencing a cybersecurity breach that would negatively impact the business operations within that organisation. Thus, the successful design, implementation, testing and maintenance of at least half, of these recommendations will yield higher successful results compared to the meagre implementation of all eleven recommendations to complete a checklist of defences against cybersecurity threats or regulatory audits.

\section{References}

[1] Frederico Araujo and Teryl Taylor. 2020. Improving cybersecurity hygiene through JIT patching. In Proceedings of the 28th ACM foint Meeting on European Software Engineering Conference and Symposium on the Foundations of Software Engineering. 1421-1432.

[2] Gabriel Bassett. 2020. https://enterprise.verizon.com/content/ verizonenterprise/us/en/index/resources/reports/2020-data-breachinvestigations-report.pdf

[3] Ebrima N Ceesay. 2008. Mitigating phishing attacks: a detection, response and evaluation framework. University of California, Davis.

[4] CityPowerJhb. 2020. https://twitter.com/CityPowerJhb/status/ 1154277777950093313

[5] Joseph Cox. 2020. https://www.vice.com/en_us/article/jgxd3d/twitterinsider-access-panel-account-hacks-biden-uber-bezos

[6] MN Dazahra, F Elmariami, A Belfqih, and Jamal Boukherouaa. 2018. Defense-in-depth Cybersecurity for Smart Substations. International Journal of Electrical \& Computer Engineering (2088-8708) 8, 6 (2018).
[7] Domesticus. [n.d.]. https://www.enigmasoftware.com/w32virutcfremoval/

[8] Garmin. 2020. https://newsroom.garmin.com/newsroom/pressrelease-details/2020/Garmin-issues-statement-on-recentoutage/default.aspx

[9] GoldSparrow. [n.d.]. https://www.enigmasoftware.com/dealbeaverremoval/

[10] Engin Kirda, Christopher Kruegel, Greg Banks, Giovanni Vigna, and Richard Kemmerer. 2006. Behavior-based Spyware Detection.. In Usenix Security Symposium. 694.

[11] Jian-hua Li. 2018. Cyber security meets artificial intelligence: a survey. Frontiers of Information Technology \& Electronic Engineering 19, 12 (2018), 1462-1474.

[12] Microsoft. 2019. https://docs.microsoft.com/en-us/windowsserver/identity/securing-privileged-access/securing-privilegedaccess-reference-material

[13] Samuel Moses and Dale C Rowe. 2016. Physical security and cybersecurity: Reducing risk by enhancing physical security posture through multi-factor authentication and other techniques. International fournal for Information Security Research (IfISR) 6, 2 (2016).

[14] Admire Moyo. 2019. https://www.itweb.co.za/content/ KBpdgvpPG8lvLEew

[15] Muyowa Mutemwa, Jabu Mtsweni, and Lukhanyo Zimba. 2018. Integrating a security operations centre with an organization's existing procedures, policies and information technology systems. In 2018 International Conference on Intelligent and Innovative Computing Applications (ICONIC). IEEE, 1-6.

[16] Stelian Pilici. 2019. https://malwaretips.com/blogs/remove-heuradvml-b/

[17] PricewaterhouseCoopers. 2020. https://www.pwc.co.za/en/pressroom/23rd-annual-ceo-survey-2020.html

[18] PricewaterhouseCoopers. 2020. https://www.pwc.co.za/en/pressroom/global-economic-crime-and-fraud-survey-2020.html

[19] Jan Rüth, Torsten Zimmermann, Konrad Wolsing, and Oliver Hohlfeld. 2018. Digging into browser-based crypto mining. In Proceedings of the Internet Measurement Conference 2018. 70-76.

[20] SABRIC. 2019. https://www.sabric.co.za/media-and-news/pressreleases/south-african-banks-resilient-in-the-face-of-latest-ddosattacks/

[21] Bisma Shah. 2017. Cisco Umbrella: A Cloud-Based Secure Internet Gateway (SIG) On and Off Network. International fournal of Advanced Research in Computer Science 8, 2 (2017).

[22] Sophos. [n.d.]. https://www.sophos.com/en-us/threat-center/threatanalyses/viruses-and-spyware/CXmail OleDI-AD/detailedanalysis.aspx

[23] Sophos. 2020. https://community.sophos.com/kb/en-us/124699

[24] Javier Martínez Torres, Carla Iglesias Comesaña, and Paulino J GarciaNieto. 2019. Machine learning techniques applied to cybersecurity. International fournal of Machine Learning and Cybernetics 10, 10 (2019), 2823-2836.

[25] Twitter. 2020. https://blog.twitter.com/en_us/topics/company/2020/ an-update-on-our-security-incident.html

[26] Staff Writer. 2019. https://businesstech.co.za/news/energy/331247/ city-power-hit-by-ransomware-virus/

[27] Roman V Yampolskiy and MS Spellchecker. 2016. Artificial intelligence safety and cybersecurity: A timeline of AI failures. arXiv preprint arXiv:1610.07997 (2016).

[28] ZulaZuza. [n.d.]. https://www.enigmasoftware.com/trojangen2removal/ 